\title{
The WEBT campaign to observe AO 0235+16 in the 2003-2004 observing season ${ }^{\star}$
}

\section{Results from radio-to-optical monitoring and XMM-Newton observations}

\author{
C. M. Raiteri ${ }^{1}$, M. Villata ${ }^{1}$, M. A. Ibrahimov ${ }^{2}$, V. M. Larionov ${ }^{3,4}$, M. Kadler ${ }^{5}$, H .D. Aller ${ }^{6}$, M. F. Aller ${ }^{6}$,
} Y. Y. Kovalev ${ }^{7,8 \star \star}$, L. Lanteri ${ }^{1}$, K. Nilsson ${ }^{9}$, I. E. Papadakis ${ }^{10,11}$, T. Pursimo ${ }^{12}$, G. E. Romero ${ }^{13}$, H. Teräsranta ${ }^{14}$ M. Tornikoski ${ }^{14}$, A. A. Arkharov ${ }^{15}$, D. Barnaby ${ }^{16}$, A. Berdyugin ${ }^{9}$, M. Böttcher ${ }^{17}$, K. Byckling ${ }^{18}$, M. T. Carini ${ }^{16}$, D. Carosati ${ }^{19}$, S. A. Cellone ${ }^{20}$, S. Ciprini ${ }^{9}$, J. A. Combi ${ }^{13,21}$, S. Crapanzano ${ }^{1}$, R. Crowe ${ }^{22}$, A. Di Paola ${ }^{23}$, M. Dolci ${ }^{24}$, L. Fuhrmann ${ }^{1,5,25}$, M. Gu ${ }^{26}$, V. A. Hagen-Thorn ${ }^{3,4}$, P. Hakala ${ }^{18}$, V. Impellizzeri ${ }^{5}$, S. Jorstad ${ }^{27}$, J. Kerp ${ }^{5}$, G. N. Kimeridze ${ }^{28}$, Yu. A. Kovalev ${ }^{8}$, A. Kraus ${ }^{5}$, T. P. Krichbaum ${ }^{5}$, O. M. Kurtanidze ${ }^{28}$, A. Lähteenmäki ${ }^{14}$,

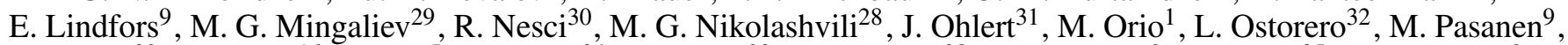
A. Pati ${ }^{33}$, C. Poteet ${ }^{16}$, E. Ros ${ }^{5}$, J. A. Ros ${ }^{34}$, P. Shastri ${ }^{33}$, L. A. Sigua ${ }^{28}$, A. Sillanpääa ${ }^{9}$ N. Smith ${ }^{35}$, L. O. Takalo ${ }^{9}$, G. Tosti ${ }^{25}$, A. Vasileva ${ }^{3}$, S. J. Wagner ${ }^{32}$, R. Walters ${ }^{16}$, J. R. Webb ${ }^{36}$, W. Wills ${ }^{16}$, A. Witzel ${ }^{5}$, and E. Xilouris ${ }^{37}$

${ }^{1}$ INAF - Osservatorio Astronomico di Torino, Italy, e-mail: raiteri@to.astro.it ${ }^{2}$ Ulugh Beg Astronomical Inst., Uzbekistan ${ }^{3}$ Astronomical Inst., St.-Petersburg State Univ., Russia ${ }^{4}$ Isaac Newton Inst. of Chile, St. Petersburg Branch ${ }^{5}$ Max-Planck-Institut für Radioastronomie, Germany ${ }^{6}$ Dept. of Astronomy, Univ. of Michigan, USA ${ }^{7}$ National Radio Astronomy Observatory, Green Bank, USA ${ }^{8}$ Astro Space Center of Lebedev Physical Inst., Russia ${ }^{9}$ Tuorla Astronomical Observatory, Finland ${ }^{10}$ IESL, FORTH, Greece ${ }^{11}$ Physics Dept., Univ. of Crete, Greece ${ }^{12}$ Nordic Optical Telescope, Santa Cruz de La Palma, Spain ${ }^{13}$ Inst. Argentino de Radioastronomía, Argentina ${ }^{14}$ Metsähovi Radio Observatory, Helsinki Univ. of Technology, Finland ${ }^{15}$ Pulkovo Observatory, Russia ${ }^{16}$ Dept. of Physics \& Astronomy, Western Kentucky Univ., USA ${ }^{17}$ Dept. of Physics and Astronomy, Ohio Univ., USA ${ }^{18}$ Observatory, Univ. of Helsinki, Finland ${ }^{19}$ Armenzano Astronomical Observatory, Italy ${ }^{20}$ Facultad de Ciencias Astronómicas y Geofísicas, Argentina ${ }^{21}$ Dept. de Física, Escuela Politécnica Superior, Univ. de Jaén, Spain ${ }^{22}$ Dept. of Physics \& Astronomy, Univ. of Hawaii, USA ${ }^{23}$ INAF - Osservatorio Astronomico di Roma, Italy ${ }^{24}$ INAF - Osservatorio Astronomico di Collurania Teramo, Italy ${ }^{25}$ Osservatorio Astronomico, Univ. di Perugia, Italy ${ }^{26}$ Korea Astronomy Observatory, Korea ${ }^{27}$ Inst. for Astrophysical Research, Boston Univ., USA ${ }^{28}$ Abastumani Observatory, Georgia ${ }^{29}$ Special Astrophysical Observatory, Karachaevo-Cherkessia, Russia ${ }^{30}$ Dipartimento di Fisica, Univ. di Roma "La Sapienza", Italy ${ }^{31}$ Michael Adrian Observatory, Trebur, Germany ${ }^{32}$ Landessternwarte Heidelberg, Germany ${ }^{33}$ Indian Inst. of Astrophysics, Bangalore, India ${ }^{34}$ Agrupació Astronòmica de Sabadell, Spain ${ }^{35}$ Dept. of Applied Physics \& Instrumentation, Cork Inst. of Technology, Ireland ${ }^{36}$ SARA Observatory, Florida International Univ., USA ${ }^{37}$ Inst. of Astronomy and Astrophysics, National Observatory of Athens, Greece

Received 17 December 2004 / Accepted 17 February 2005

\begin{abstract}
A multiwavelength campaign to observe the BL Lac object AO 0235+16 $(z=0.94)$ was set up by the Whole Earth Blazar Telescope (WEBT) collaboration during the observing seasons 2003-2004 and 2004-2005, involving radio, near-IR and optical photometric monitoring, VLBA monitoring, optical spectral monitoring, and three pointings by the XMM-Newton satellite. Here we report on the results of the first season, which involved the participation of 24 optical and near-IR telescopes and 4 radio telescopes, as well as the first XMM-Newton pointing, which occurred on January 18-19, 2004. Unpublished data from previous epochs were also collected (from 5 optical-NIR and 3 radio telescopes), in order to fill the gap between the end of the period presented in Raiteri et al. (2001) and the start of the WEBT campaign. The contribution of the southern AGN, 2 arcsec distant from the source, is taken into account. It is found to especially affect the blue part of the optical spectrum when the source is faint. In the optical and near-IR the source has been very active in the last 3 years, although it has been rather faint most of the time, with noticeable variations of more than a magnitude over a few days. In contrast, in the radio bands it appears to have been "quiescent" since early 2000. The major radio (and optical) outburst predicted to peak around February-March 2004 (with a six month uncertainty) has not occurred yet. When comparing our results with the historical light
\end{abstract}

\footnotetext{
* For questions regarding the availability of the data presented in this paper, please contact the WEBT President Massimo Villata (villata@to.astro.it).

$\star \star$ Jansky Fellow.
} 
curves, two different behaviours seem to characterize the optical outbursts: only the major events present a radio counterpart. The X-ray spectra obtained by the three EPIC detectors are well fitted by a power law with extra-absorption at $z=0.524$; the energy index in the $0.2-10 \mathrm{keV}$ range is well constrained: $\alpha=0.645 \pm 0.028$ and the $1 \mathrm{keV}$ flux density is $0.311 \pm 0.008 \mu \mathrm{Jy}$. The analysis of the X-ray light curves reveals that no significant variations occurred during the pointing. In contrast, simultaneous dense radio monitoring with the $100 \mathrm{~m}$ telescope at Effelsberg shows a $\sim 2-3 \%$ flux decrease in 6-7 h, which, if intrinsic, would imply a brightness temperature well above the Compton limit and hence a lower limit to the Doppler factor $\delta \gtrsim 46$. We construct the broad-band spectral energy distribution of January 18-19, 2004 with simultaneous radio data from Effelsberg, optical data from the Nordic Optical Telescope (NOT), optical-UV data from the Optical Monitor onboard XMMNewton, and X-ray data by the EPIC instruments. Particular care is taken to correct data for extinction due to both the Milky Way and the $z=0.524$ absorber. The resulting SED suggests the existence of a bump in the UV spectral region.

Key words. galaxies: active - BL Lacertae objects: general - BL Lacertae objects: individual: AO 0235+16

\section{Introduction}

BL Lac objects belong to the blazar class of active galactic nuclei (AGNs). They show variability at all wavelengths, from the radio to the $\gamma$-ray band, on a variety of time scales. In general, slow oscillations of the flux mean level on time scales of weeks to years have been observed, based on which fast flares lasting as little as a few hours are superposed. The commonly accepted scenario for blazar emission assumes a black hole surrounded by an accretion disc, feeding a plasma jet which is oriented close to the line of sight. This geometrical configuration can explain the apparent superluminal motion of relativistically moving plasma down the jet which is often derived from the analysis of radio maps. Moreover, relativistic Doppler effects can, at least in part, explain the extremely high brightness temperature which otherwise would result in the so-called Compton catastrophe.

The best way to study the source flux variability and to understand the corresponding physical processes is the simultaneous observation across the whole accessible electromagnetic spectrum. Indeed, it is commonly accepted that the observed lower-energy flux (from the radio band to the UV-X-ray one) is due to synchrotron emission from the plasma jet, while the higher-energy one (up to $\mathrm{GeV}-\mathrm{TeV}$ energies) is thought to be produced by inverse-Compton scattering of soft photons off the same relativistic electrons producing the synchrotron radiation. It goes without saying that the high-energy flux should thus be correlated with the low-energy one, but the relative variability amplitude and the amount of time lag expected depend on the particular model adopted.

Analysis of the long-term radio and optical light curves of $\mathrm{AO} 0235+16$ led to the conclusion that the main radio outbursts have optical counterparts, and that they repeat every $5.7 \pm 0.5$ years (Raiteri et al. 2001). This was interpreted in terms of geometrical effects due to the possible presence of a binary black hole system (Romero et al. 2003; Ostorero et al. 2004). The next outburst was predicted to peak around February-March 2004, and a multiwavelength radio-NIR-optical campaign has been organized by the Whole Earth Blazar Telescope (WEBT; http://www.to.astro.it/blazars/webt/; e.g. Villata et al. 2000, 2002, 2004a,b) collaboration to follow the source behaviour. The WEBT campaign began in summer 2003, and was planned to continue until spring 2005. During this longterm campaign three pointings by the XMM-Newton satellite were granted in order to compare the behaviour of the low-energy emission with the high-energy one. The XMM-Newton observations were scheduled on January 18-19 and August 2, 2004, and January 28, 2005. We know from previous observations by X-ray satellites (Einstein et al. 1990; EXOSAT, Ghosh \& Soundararajaperumal 1995; ROSAT, Madejski et al. 1996; Comastri et al. 1997; ASCA, Madejski et al. 1996; Junkkarinen et al. 2004; RXTE, Webb et al. 2000; BeppoSAX, Padovani et al. 2004) that both the X-ray flux and spectral index can be very variable.

The variability of AO $0235+16$ on short time scales is also of special interest. At radio wavelengths several flatspectrum radio quasars and BL Lacs are known to exhibit rapid variability (Witzel et al. 1986; Heeschen et al. 1987; Kedziora-Chudczer et al. 2001; Lovell et al. 2003; Kraus et al. 2003) on time scales of days to hours (intraday variability, IDV). The cause of the variations seen in these sources is currently controversial, with claims being made for either a source-intrinsic (e.g. shocks in the emitting jet) or extrinsic origin (interstellar scintillation or gravitational microlensing). Interstellar scintillation (ISS) may play an important role in the cm-radio regime (Jauncey \& Macquart 2001; Rickett et al. 2001; Dennet-Thorpe \& de Bruyn 2002), otherwise intrinsic variations may require extremely high Doppler boosting $(\delta \sim 100)$ or special source geometries in order to prevent the inverse-Compton catastrophe (see e.g. Wagner \& Witzel 1995; Wagner et al. 1996). Since ISS cannot explain correlated IDV over a wide range of the electromagnetic spectrum, the detection of broad-band correlations would directly rule out ISS as sole explanation for radio IDV and would favour a source intrinsic contribution to the IDV pattern.

AO $0235+16$ has shown IDV over a wide range of the electromagnetic spectrum, from the radio to the optical band (Takalo et al. 1992; Heidt \& Wagner 1996; Noble \& Miller 1996; Romero et al. 1997; Kraus et al. 1999; Romero et al. 2000; Raiteri et al. 2001). Moreover, hints for the existence of very high Doppler factors come from a variety of other observational studies (Fujisawa et al. 1999; Frey et al. 2000; Jorstad et al. 2001), thus making this blazar an ideal candidate for the detection of correlated broad-band IDV. XMM-Newton has already revealed fast X-ray variability of AO $0235+16$ (Kadler et al. 2005). Consequently, one of the aims of the WEBT campaign was to search for radio-NIR-optical-X-ray correlated fast variations during the XMM-Newton pointings, with the support of dense radio monitoring by the $100 \mathrm{~m}$ radio telescope at Effelsberg. 
Side by side with the WEBT campaign, spectroscopic monitoring of the source in the optical band with both the Telescopio Nazionale Galileo (TNG) and the ESO's New Technology Telescope (NTT) has been carried out (Raiteri et al. 2005). 15 VLBA epochs have been awarded to monitor the source structure variability during the WEBT campaign (Wiik et al. 2005).

In this paper we present results obtained during the first observing season of the campaign, from July 1, 2003 to April 2004, including the first XMM-Newton pointing of January 18-19, 2004. We also collected data taken before the start of the campaign, back to the last data published by Raiteri et al. (2001), in order to reconstruct the source behaviour in between. Other papers will follow, containing new results and more detailed analyses.

The long-term optical, NIR and radio monitoring data obtained by the WEBT during the 2003-2004 observing season, along with the data filling the gap between this WEBT campaign and the data published by Raiteri et al. (2001), are presented in Sect. 2. The multiwavelength observations performed during the XMM-Newton pointing of January 18-19, 2004 are analysed in Sect. 3. In Sect. 4 we discuss the problem of extinction and construct the broad-band spectral energy distribution of $\mathrm{AO} 0235+16$ during the XMM-Newton pointing. Section 5 contains some results of the cross-correlation analysis. Conclusions are drawn in Sect. 6.

\section{Long-term observations by the WEBT}

The WEBT campaign was started on July 1, 2003; the last data presented here were taken in April 2004. However, for the present work we collected data from previous epochs in order to fill the gap between the start of the WEBT campaign and the last data published in Raiteri et al. (2001), and to enrich the historical light curves.

\subsection{Optical and near-IR data}

Tables 1 and 2 show the lists of the optical and near-IR telescopes participating in the WEBT campaign (and prior data collection) ordered by longitude ${ }^{1}$. In the tables Col. 1 contains the name of the observatory and its location; Col. 2 gives the telescope diameter in $\mathrm{cm}$; Cols. 3-7 report the number of observations done in the UBVRI and JHK bands; Col. 8 shows the total number of observations performed by each telescope in those bands. Data in brackets indicate the number of points remaining after the "cleaning" procedure described below and which are shown in the light curves. As one can see in the last row, the total number of observations collected is 2509 in the optical and 385 in the near-IR.

Data were collected as instrumental magnitudes of the source and comparison stars in the same field.

\footnotetext{
1 One of the main advantages of WEBT is to have observers spread out at different longitudes. Hence, the observing task can move from east to west allowing us in principle to obtain continuous monitoring over $24 \mathrm{~h}$.
}

The source was calibrated preferentially with respect to Stars 123 (UBVRI photometry by Smith et al. 1985). However, since the source was rather faint, in some cases long exposure times produced saturation of Star 2 (and Star 3), so that it was necessary to include other reference stars for the calibration. Hence, we sometimes also used Stars 6 and $\mathrm{C} 1$ calibrated in VRI by Fiorucci et al. (1998). We made several checks to test the variation of the source magnitude value when adopting different choices of reference stars for the calibration: in general, we saw that the variation is within a few hundredths of a mag, which is usually inside the errors.

In order to construct meaningful light curves, the observations reported in Table 1 were carefully analysed and unreliable data discarded. This was done by zooming into subsequent very short periods of time and inspecting all the light curves at the same time. The same trend in all bands was required within errors; bad points were eliminated. In the case of noisy intranight datasets from the same telescope, some binning over time intervals not exceeding $20 \mathrm{~min}$ was performed. In general, data affected by errors larger than 0.2 mag were not included.

Moreover, we investigated the possibility that our photometric measurements of AO $0235+16$ were affected by the contribution of objects very close to the source. The complex environment of the source was studied by various authors (Yanny et al. 1989; Burbidge et al. 1996; Nilsson et al. 1996); they noticed that there are a few galaxies at redshift of 0.524 in the source surroundings, and two of them are very close to AO $0235+16$. One galaxy, probably a normal spiral, is $1.3 \mathrm{arcsec}$ to the east, but it is very faint and thus does not affect the source photometry, even when the source is faint. The object about 2 arcsec to the south is more problematic, since it is brighter than the former one, and it is also known to be an AGN, possibly interacting with the eastern galaxy. The photometric contribution of this southern object (hereafter named ELISA: elusive intervening southern AGN) can affect the source magnitude when it is very faint, especially in the bluer part of the spectrum. This increasing contribution towards the ultraviolet is mainly due to the fact that the AO $0235+16$ spectrum is much steeper than that of ELISA, since AO 0235+16 is strongly absorbed in these bands (see Sect. 4). We thus estimated the magnitude of ELISA from the Mt. Maidanak and Hanle frames, where it is often clearly resolved. There is some dispersion in the data, and it is not possible to recognize any clear variability trend in the period 2000-2004. The best estimates are: $U=20.8, B=21.4, V=20.9, R=20.5, I=19.9$. These values are in fair agreement with $R=20.4 \pm 0.1$, $R-I=0.7 \pm 0.2$ given by Nilsson et al. (1996), and with $m_{450}=21.40 \pm 0.01$ estimated by Burbidge et al. (1996). We then converted the AO 0235+16 magnitudes into fluxes, subtracted the ELISA contribution, and then converted again into magnitudes.

In Fig. 1 we present the UBVRI light curves obtained during the WEBT campaign, after applying both the cleaning and ELISA-subtraction procedures described above. These are the light curves that will be used for the subsequent analysis. Data from different telescopes are plotted with different colours and symbols in order to be able to distinguish the single 
Table 1. List of participating optical observatories by longitude and number of observations performed in the various bands.

\begin{tabular}{|c|c|c|c|c|c|c|c|}
\hline Observatory & $d(\mathrm{~cm})$ & $N_{U}$ & $N_{B}$ & $N_{V}$ & $\overline{N_{R}}$ & $N_{I}$ & $\overline{N_{\text {tot }}}$ \\
\hline IAO Hanle, India & 200 & $8(6)$ & $7(6)$ & $12(10)$ & $8(8)$ & $14(13)$ & $49(43)$ \\
\hline Mt. Maidanak, Uzbekistan & 150 & $59(42)$ & $336(311)$ & $112(107)$ & $450(417)$ & 237 (219) & 1194 (1096) \\
\hline Abastumani, Georgia (FSU) & 70 & 0 & 0 & 0 & $41(32)$ & 0 & $41(32)$ \\
\hline Crimean, Ukraine & 70 & 0 & 0 & $17(16)$ & $19(18)$ & $19(19)$ & $55(53)$ \\
\hline Skinakas, Crete & 130 & 0 & $24(17)$ & $27(23)$ & $28(27)$ & $26(25)$ & $105(92)$ \\
\hline Tuorla, Finland & 103 & 0 & 0 & 0 & $44(17)$ & 0 & $44(17)$ \\
\hline Vallinfreda, Italy & 50 & 0 & 0 & 0 & $1(1)$ & 0 & $1(1)$ \\
\hline Armenzano, Italy & 40 & 0 & $14(12)$ & $28(18)$ & $41(32)$ & $29(26)$ & $112(88)$ \\
\hline Perugia, Italy & 40 & 0 & 0 & $2(0)$ & $4(1)$ & $4(4)$ & $10(5)$ \\
\hline Michael Adrian, Germany & 120 & 0 & 0 & 0 & $7(5)$ & 0 & $7(5)$ \\
\hline Greve, Italy & 32 & 0 & 0 & 0 & $1(1)$ & 0 & $1(1)$ \\
\hline Torino, Italy & 105 & 0 & $6(2)$ & $12(11)$ & $132(102)$ & $83(62)$ & $233(177)$ \\
\hline Heidelberg, Germany & 70 & 0 & 0 & 0 & $4(2)$ & $1(0)$ & $5(2)$ \\
\hline Calar Alto, Spain & 220 & 0 & 0 & 0 & $101(15)$ & 0 & $101(15)$ \\
\hline Sabadell, Spain & 50 & 0 & 0 & 0 & $90(1)$ & 0 & $90(1)$ \\
\hline Roque (KVA), La Palma & 35 & 0 & 0 & 0 & $180(95)$ & 0 & $180(95)$ \\
\hline Roque (NOT), La Palma & 256 & $12(7)$ & $14(13)$ & $14(13)$ & $23(23)$ & $17(15)$ & $80(71)$ \\
\hline Roque (TNG), La Palma & 358 & 0 & 0 & 0 & $2(2)$ & 0 & $2(2)$ \\
\hline CASLEO, Argentina & 215 & 0 & 0 & $55(55)$ & $59(59)$ & 0 & $114(114)$ \\
\hline Bell, Kentucky & 60 & 0 & 0 & 0 & $10(10)$ & 0 & $10(10)$ \\
\hline Kitt Peak (SARA), Arizona & 90 & 0 & 0 & $3(2)$ & $30(19)$ & $3(3)$ & $36(24)$ \\
\hline Mt. Lemmon, Arizona & 100 & 0 & $2(0)$ & $5(2)$ & $8(5)$ & $5(5)$ & $20(12)$ \\
\hline Lowell (Perkins), Arizona & 180 & 0 & $1(1)$ & $3(2)$ & $4(4)$ & $3(3)$ & $11(10)$ \\
\hline Mauna Kea, Hawaii & 60 & 0 & $3(0)$ & $4(1)$ & $0(0)$ & $1(1)$ & $8(2)$ \\
\hline Total & & $79(55)$ & $407(362)$ & $294(260)$ & 1287 (896) & $442(395)$ & 2509 (1968) \\
\hline
\end{tabular}

Table 2. List of participating near-IR observatories by longitude and number of observations performed in the various bands.

\begin{tabular}{lc|ccc|c}
\hline \hline Observatory & $d(\mathrm{~cm})$ & $N_{J}$ & $N_{H}$ & $N_{K}$ & $N_{\text {tot }}$ \\
\hline Campo Imperatore, Italy & 110 & $82(82)$ & $81(81)$ & $88(87)$ & $251(250)$ \\
TIRGO, Switzerland & 150 & $59(1)$ & $1(1)$ & $55(1)$ & $115(3)$ \\
Roque (NOT), La Palma & 256 & $6(6)$ & $5(5)$ & $8(8)$ & $19(19)$ \\
\hline Total & & $147(89)$ & $87(87)$ & $151(96)$ & $385(272)$ \\
\hline
\end{tabular}

contributions as well as to appreciate how well the datasets agree with one another.

The source was in a rather faint state, but was undergoing noticeable variability, with a total variation of about 2 mag in the best sampled $R$ band, and changes of about 1 mag on time scales of about a week.

Average colour indices (and standard deviations) obtained by coupling data acquired by the same telescope within $20 \mathrm{~min}$ are: $B-R=1.72(0.12), V-R=0.76(0.10), R-I=0.99(0.09)$. These averages are obtained from 223,151 and 296 indices, respectively.

Near-IR observations during the WEBT campaign have been carried out with the $110 \mathrm{~cm}$ telescope at Campo Imperatore and the $256 \mathrm{~cm}$ Nordic Optical Telescope (NOT). The source magnitude was calibrated with the
González-Pérez et al. (2001) photometry. The contribution from ELISA in this frequency regime is negligible.

Figure 2 shows the $J H K$ light curves compared with the $R$-band one. The near-IR light curves are generally undersampled to allow a detailed statement about a correspondence with the optical data, but where sufficient sampling has been achieved, they indicate that a close correspondence exists, as expected. Average colour indices (and standard deviations) obtained by coupling data acquired by the same telescope within 20 min are: $J-K=2.12(0.09), H-K=1.00$ (0.07). A rough estimate of optical-NIR colour indices can be obtained by coupling data within $60 \mathrm{~min}: R-J=2.50(0.23), R-H=3.60$ (0.29), $R-K=4.58(0.34)$.

$J H K$ data before the campaign were obtained on December 18, $2002(\mathrm{JD}=2452627)$ with the $150 \mathrm{~cm}$ Italian 


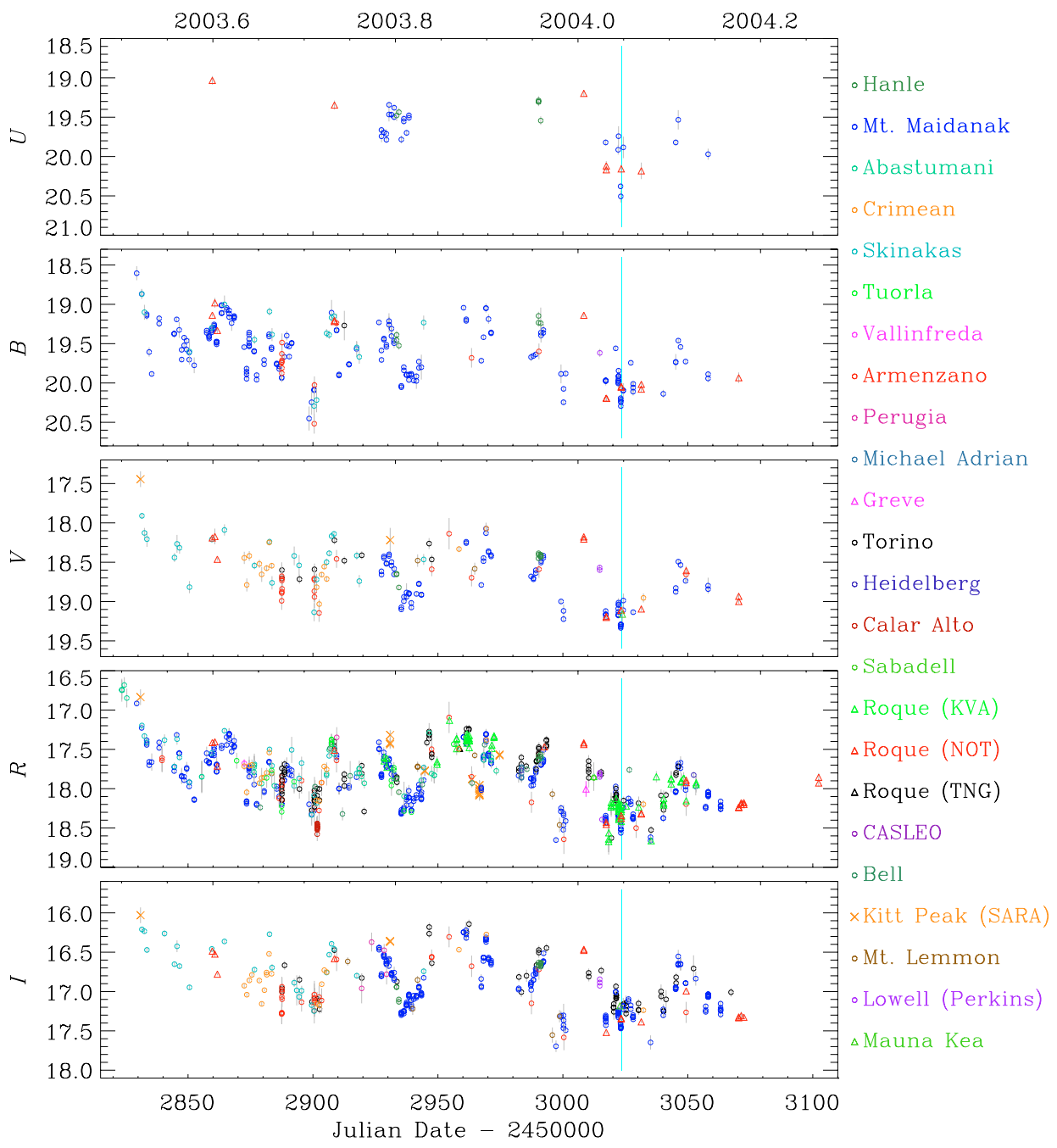

Fig. 1. UBVRI light curves of AO 0235+16 during the observing season 2003-2004; the XMM-Newton pointing of January 18-19 is marked by the vertical (cyan) line at JD $=2453023$. The ELISA contribution has been subtracted as explained in the text.

national telescope TIRGO, at Gornergrat, Switzerland. These data yield average values of $J=15.28 \pm 0.06, H=14.36 \pm$ $0.10, K=13.17 \pm 0.08$.

\subsection{Intraday variability}

On very short time scales, the source displayed clear variability in various occasions. As an example, well-sampled intranight light curves in $V$ and $R$ bands were obtained from November 10 to 14,2001 , with the $215 \mathrm{~cm}$ telescope of the CASLEO, Argentina. The $R$-band light curve is shown in Fig. 3, with the same calibration and ELISA flux subtraction adopted for the data in Fig. 1. Clear intranight trends are recognizable on November 10, 12, and 13, with smooth variations of about $0.1 \mathrm{mag}$ in a few hours. On internight time scales, variations as fast as $\sim 0.5$ mag in 1-2 days were registered.

Although this behaviour is not as extreme as observed in other occasions, such as the $\sim 1.2$ mag brightening in one day observed by Romero et al. (2000) in November 1999, or similar fast variations detected in the period 1997-1999
(Raiteri et al. 2001), nonetheless it reveals that the source can be very active even during faint states.

\subsection{Radio data}

Long-term radio observations during the WEBT campaign were performed with the $14 \mathrm{~m}$ antenna of the Metsähovi Radio Observatory in Finland, the $26 \mathrm{~m}$ paraboloid of the University of Michigan Radio Astronomy Observatory (UMRAO) in the United States, and the $600 \mathrm{~m}$ ring transit radio telescope RATAN-600 of the Russian Academy of Sciences in Russia. Details on the observing and data reduction procedures can be found e.g. in Teräsranta et al. (1998), Aller et al. (1999), and Kovalev et al. (1999a).

A comparison among optical magnitudes in the bestsampled $R$ band and radio fluxes at different frequencies is shown in Fig. 4. Data at 37 and $22 \mathrm{GHz}$ are from the Metsähovi Radio Observatory; data at $14.5,8.0$, and $4.8 \mathrm{GHz}$ are from UMRAO; those at $21.7,11.1,7.7,3.9$, and $2.3 \mathrm{GHz}$ are from RATAN. The two datasets at $37 \mathrm{GHz}$ from Metsähovi have been obtained by two different teams following slightly 


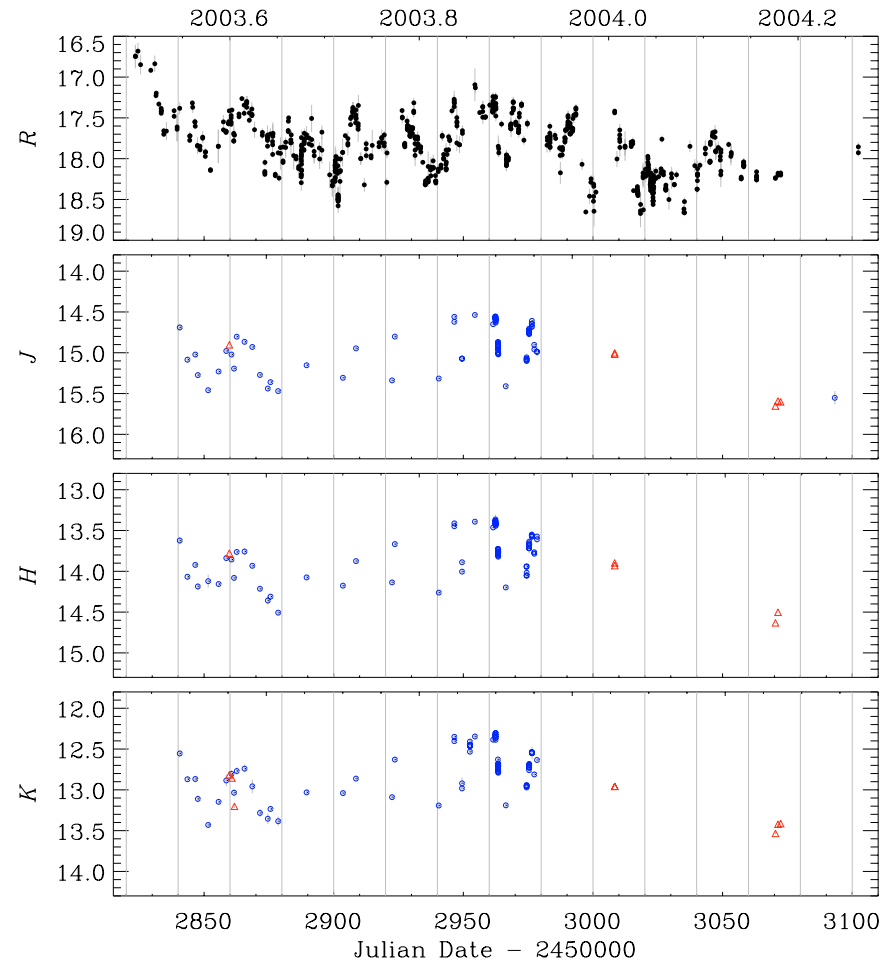

Fig. 2. JHK light curves of AO 0235+16 during the 2003-2004 observing season compared to the ELISA-subtracted $R$-band one (top). NIR data from Campo Imperatore are shown by (blue) circles, while (red) triangles indicate NIR data from the NOT.

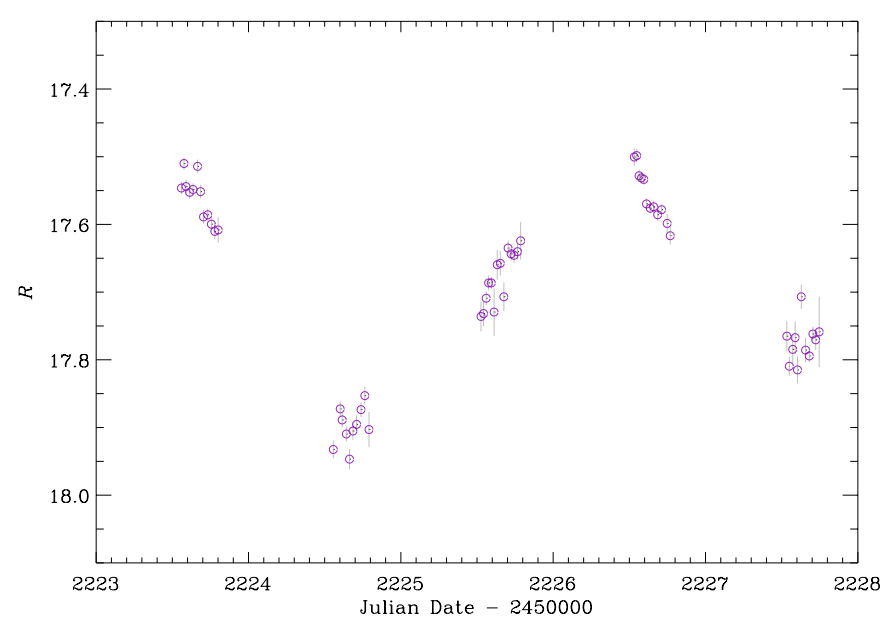

Fig. 3. $R$-band light curve of AO 0235+16 during November 10-14, 2001; data acquired with the $215 \mathrm{~cm}$ telescope of the CASLEO, Argentina.

different procedures. Further data at 4.85 and $10.45 \mathrm{GHz}$ were taken with the $100 \mathrm{~m}$ radio telescope at Effelsberg during the XMM-Newton pointing of January 18-19, 2004 (see Sect. 3.2, for more details). ELISA is not a radio emitting object, so no correction for its contribution is required.

The figure clearly shows a decreasing variability amplitude accompanied by an increasing base level when going from the higher to the lower radio frequencies. In general, after an initial small bump and a subsequent lower state, a quasi-linear trend seems to characterize the radio light curves starting from JD 2452970 , the slope of which decreases with increasing wavelength, from $4.0 \times 10^{-3}$ at $37 \mathrm{GHz}$ to $-0.3 \times 10^{-3}$ at $5 \mathrm{GHz}$. A correlation with the optical data, if it exists, is not obvious.

The analysis of the long-term radio and optical light curves led Raiteri et al. (2001) to the conclusion that the major radio outbursts repeat quasi-periodically every $\sim 5.7$ years and, when optical data are available, an optical counterpart is found immediately preceding the radio event. The next major outburst was predicted to peak in February-March 2004, with a possible window of \pm 6 months around that date. As Fig. 4 shows, no major radio or optical outburst was detected during the WEBT campaign. To make the comparison between the recent and past behaviour of the source possible, Fig. 5 displays the optical and radio light curves over a more extended time period. The period shown overlaps with that presented in Fig. 8 of Raiteri et al. (2001), and includes the last two major radio outbursts which occurred in 1992-1993 and 1998, both of them presenting an optical counterpart. The first (red) vertical line marks the end of the Raiteri et al. (2001) data; the second (green) line indicates the start of the WEBT campaign on July 1, 2003, and the third (cyan) line corresponds to the XMM-Newton pointing of January $18-19,2004$. The $R$-band data from Raiteri et al. (2001) are here complemented by data from the Hamburg Quasar Monitoring program (partially published in Schramm et al. 1994) and by data from CASLEO (Romero et al. 2002); similarly, past data from RATAN (partially published by Kovalev et al. 1999b; Kiikov et al. 2002) and data by Venturi et al. (2001) are added to the Raiteri et al. (2001) radio light curves.

There are only sparse optical data between the last data presented in the Raiteri et al. (2001) paper and the beginning of the WEBT campaign, which show a noticeable mid-term variability, with a maximum variation of $3.3 \mathrm{mag}$ in 260 days. Variations of smaller amplitude, but still noticeable, are found on shorter time scales (see also Sect. 2.2). These sparse optical data do not allow one to rule out completely the possibility that a major outburst took place in the 2000-2003.5 period, while the impressive sampling obtained during the WEBT campaign assures that no major optical outburst occured in the 2003-2004 observing season.

In contrast, the continuous radio monitoring clearly indicates that there have been no outbursts since 1998. The new data collected starting from late 2000 show that the radio flux has been low at all frequencies, even if not at the minimum levels registered in 1996-1997.

\section{The XMM-Newton pointing of January 18-19, 2004}

The X-ray Multi-Mirror Mission (XMM) - Newton satellite observed AO $0235+16$ during revolution number 0753, from January 18, 2004 at 19:06:29 UT to January 19 at 03:28:24 UT $(\mathrm{JD}=2453$ 023.29617-2453 023.64472). 

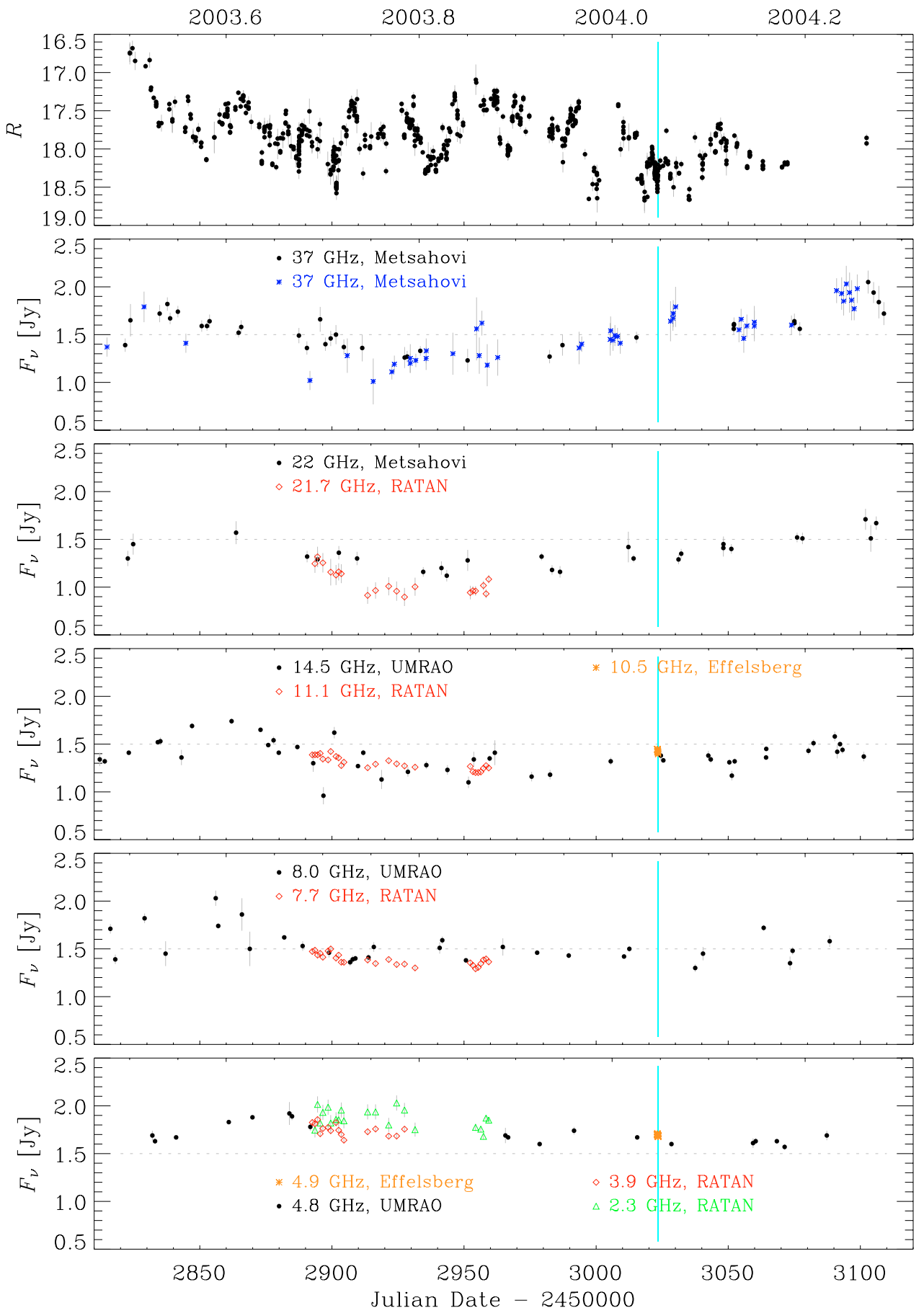

Fig. 4. ELISA-subtracted $R$-band magnitudes of AO $0235+16$ (top) and radio fluxes (Jy) at different frequencies during the WEBT campaign. The vertical (cyan) line marks the XMM-Newton pointing of January 18-19, 2004. The dotted horizontal line at $F_{v}=1.5 \mathrm{Jy}$ is drawn to guide the eye through the flux variations.

\subsection{Results from EPIC}

The European Photon Imaging Camera (EPIC) includes two types of CCD cameras: pn and MOS. There are two MOS cameras, MOS1 and MOS2, so that EPIC involves 3 detectors in total.

Since an outburst was expected, a medium filter-small window configuration was chosen for the EPIC detectors in order to avoid both possible contamination by lower-energy photons and photon pile-up. Moreover, a small window is also able to reduce the fraction of out-of-time events recorded by the EPIC-pn detector.

Data were reduced with the Science Analysis System (SAS) software version 6.0. When extracting the spectrum of both the source and the background, we included the strings "FLAG $==\varnothing$ " and "PATTERN $<=4$ " in the selection expression for all the three EPIC detectors. The first string excludes events next to the edges of the CCDs and next to bad pixels which 

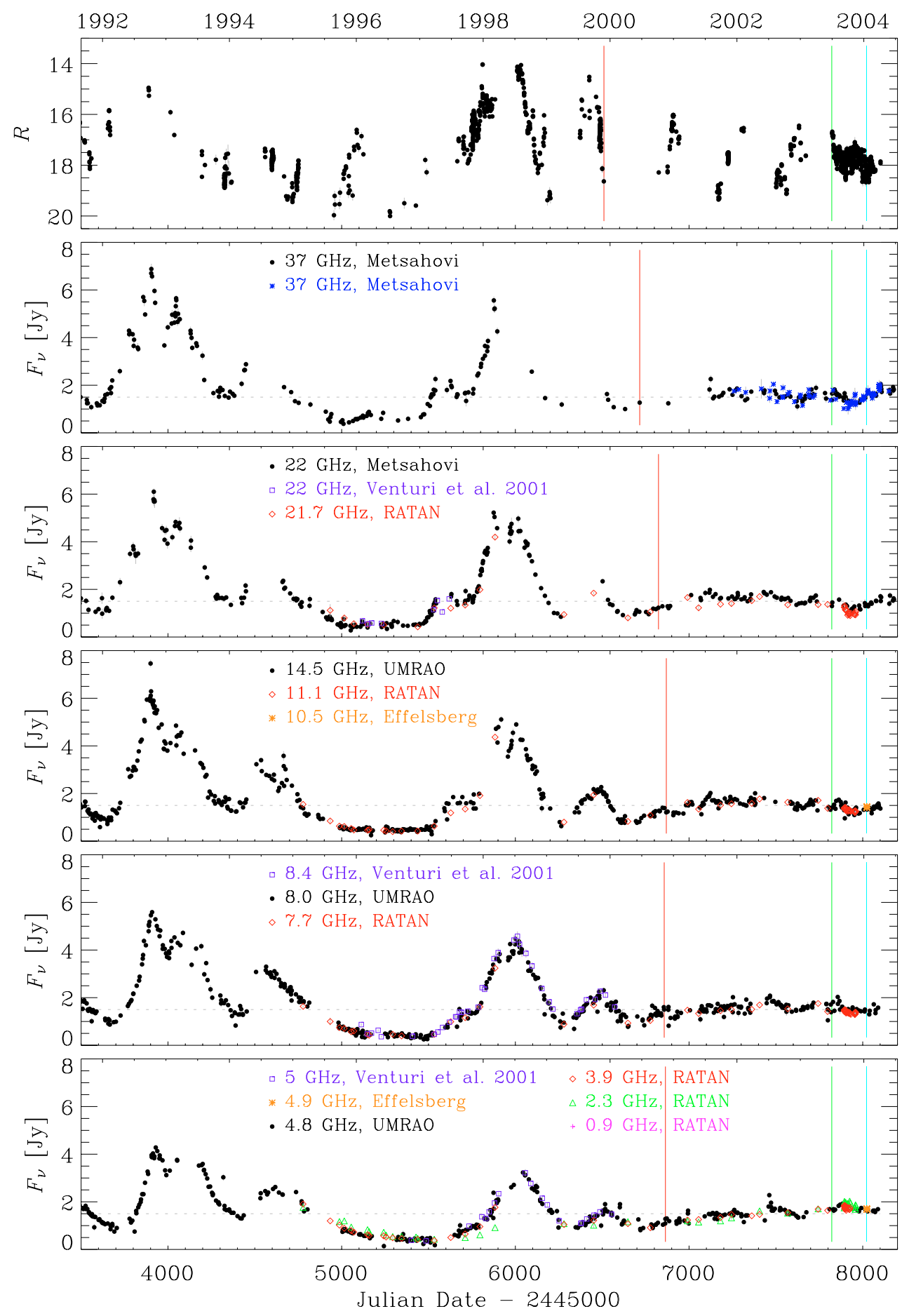

Fig. 5. ELISA-subtracted $R$-band magnitudes of AO $0235+16$ (top) compared with radio fluxes (Jy) at different frequencies; in each panel the first (red) vertical line marks the last data published by Raiteri et al. (2001); the second (green) line indicates the start of the WEBT campaign, and the third (cyan) one corresponds to the XMM-Newton pointing of January 18-19, 2004.

may have incorrect energies; the second string selects only single and double events, which have the best energy calibration.

Source spectra were extracted from a circular region with a 30 arcsec radius ${ }^{2}$. Notwithstanding the small-window configuration, we were able to extract the background spectrum on

\footnotetext{
${ }^{2}$ We examined an archive image of the AO 0235+16 field taken in August 2000 by Chandra, which has a space resolution of 0.492 arcsec, and verified that no X-ray emission was detected from
}

the same CCD of the source within circles of 20 arcsec radius in the case of MOS1 and MOS2, and 30 arcsec for pn. These background spectra, when compared with others extracted in different regions, produce the most satisfactory background subtraction.

ELISA. This could be due to intrinsic X-ray faintness, or to Compton absorption, which would favour a type 2 AGN classification of ELISA. 
The source spectra were grouped with the task "grppha" of the FTOOL package in order to have a minimum of 25 counts in each bin.

The detected net count rates are $0.1876 \pm 0.0032 \mathrm{cts} / \mathrm{s}$ for MOS1, $0.1907 \pm 0.0033 \mathrm{cts} / \mathrm{s}$ for MOS2, $0.6261 \pm$ $0.0063 \mathrm{cts} / \mathrm{s}$ for $\mathrm{pn}$.

In the following we present the results of simultaneous spectral fitting of the data from the three EPIC detectors performed with the Xspec package version 11.3.0. Only data in the channels corresponding to energy greater than $0.2 \mathrm{keV}$ were considered. Once bad channels had been ignored, the channels retained for the subsequent analysis were: 3-148 for MOS1, 6-153 for MOS2, 7-420 for pn, for a total of 708 PHA bins.

We report the results in terms of $\mathrm{HI}$ column density $N_{\mathrm{H}}$ and energy index $\alpha$, which is defined as $F_{E}=F_{0} E^{-\alpha}$.

The first approach (Model 1) was to fit a simple, absorbed power-law model to the data. The fit was good $\left(\chi^{2} / v=1.065\right.$, where $v$ is the number of degrees of freedom). The best fit was obtained with $N_{\mathrm{H}}=1.998_{-0.096}^{+0.099} \times 10^{21} \mathrm{~cm}^{-2}$ and energy in$\operatorname{dex} \alpha=0.692 \pm 0.032$. The unabsorbed $1 \mathrm{keV}$ flux density is $0.332 \pm 0.011 \mu \mathrm{Jy}$.

As expected, the value of $N_{\mathrm{H}}$ considerably exceeds the Galactic value of $7.6 \times 10^{20} \mathrm{~cm}^{-2}$ (Elvis et al. 1989), indicating extra-absorption. This extra-absorption had already been found in the previous $\mathrm{X}$-ray spectra, and is ascribed to a foreground object at $z=0.524$, which causes absorption lines in the optical spectra (Cohen et al. 1987; Nillson et al. 1996; Raiteri et al. 2005) $)^{3}$.

The next step (Model 2) was to fit the spectra with a power law with Galactic absorption plus another absorber at $z=0.524$. The fit resulted in a bit lower $\chi^{2} / v(1.019), N_{\mathrm{H}}=$ $2.450_{-0.194}^{+0.201} \times 10^{21} \mathrm{~cm}^{-2}$ at $z=0.524$, and $\alpha=0.645 \pm 0.028$ as best-fit parameters. The unabsorbed $1 \mathrm{keV}$ flux density in this case is $0.311 \pm 0.008 \mu \mathrm{Jy}$.

Although both Model 1 and Model 2 are statistically acceptable, we prefer Model 2 since it provides a plausible explanation for the absorption in excess of the Galactic value. Figure 6 shows the data from the three EPIC detectors (top) and the ratio between data and folded model (bottom) when applying Model 2.

Substituting the single power law with a broken power law does not improve the fit. A more detailed analysis of this spectrum together with other X-ray spectra of this source will be presented in a forthcoming paper (Raiteri et al. 2005).

The EPIC light curves do not show any significant variability.

\subsection{Radio-IDV observations with the Effelsberg $100 \mathrm{~m}$ telescope}

Densely sampled radio flux-density measurements with the Effelsberg $100 \mathrm{~m}$ radio telescope were performed simultaneously with the XMM-Newton observations of January 18-19, 2004 at $\lambda 2.8 \mathrm{~cm}$ and $\lambda 6 \mathrm{~cm}(10.45$ and $4.85 \mathrm{GHz})$.

\footnotetext{
3 This absorber is usually identified with the galaxy $1.3 \operatorname{arcsec}$ to the east, but a contribution from the ELISA host galaxy, claimed to be interacting with the former, cannot be ruled out.
}

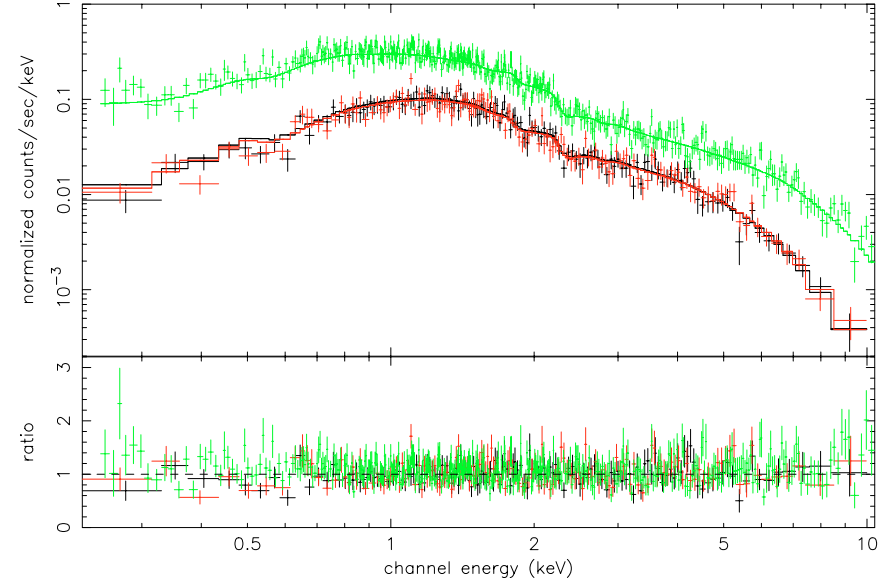

Fig. 6. Spectra from the three EPIC detectors (top) and ratio between data and folded model (bottom) when fitting the data with a powerlaw model with Galactic absorption plus absorption by the foreground galaxy at $z=0.524$. The green, black, and red points refer to the pn, MOS1, and MOS2 detectors, respectively.

On arcsecond scales, AO $0235+16$ is a compact radio source and appears point-like to the Effelsberg $100 \mathrm{~m}$ telescope. Its relative brightness of $\sim 1.7 \mathrm{Jy}$ at $\lambda 6 \mathrm{~cm}$ and $\sim 1.4 \mathrm{Jy}$ at $\lambda 2.8 \mathrm{~cm}$ in January 2004 allowed the flux-density monitoring to be performed with "cross scans" (e.g. Heeschen et al. 1987; Quirrenbach et al. 1992) over the source position. The cross scans consisted of four individual subscans in azimuthal and elevational back-and-forth direction, respectively, which in addition were used to control the telescope pointing accuracy throughout the observations. During the measurements, lefthand- and righthand-circular polarization signals were recorded and later offline combined. The flux density of AO $0235+16$ was measured 7-8 times per hour at $\lambda 6 \mathrm{~cm}$ and once per hour at $\lambda 2.8 \mathrm{~cm}$. Between the scans on the target source, the two steep-spectrum secondary calibrators, 3C 67 and $4 \mathrm{C} 08.10$, were observed, alternately. As primary flux calibrators, the sources 3C 48, 3C 161, and 3C 286 were used (see Ott et al. 1994, and references therein).

Gaussian profiles were fitted to each subscan, yielding the convolution of point-like source-brightness distribution with the telescope beam. Small residual pointing errors were corrected and the amplitudes of the subscans were averaged for each scan. The secondary calibrators were used to correct for time- and elevation-dependent antenna gain effects. The absolute calibration of the measured flux-density was achieved by scaling the registered signal from the primary calibrators to match their tabulated brightnesses. The uncertainties of the flux-density points were estimated from the residual scatter of the non-variable secondary calibrators, which dominated over the formal statistical errors. The resulting flux-density uncertainties are $\sim 0.25 \%$ at $\lambda 6 \mathrm{~cm}$ and $\sim 0.54 \%$ at $\lambda 2.8 \mathrm{~cm}$.

In Fig. 7, we show the resulting radio light curves. At both observed frequencies, AO $0235+16$ exhibited significant IDV activity on a level of $\sim 2-3 \%$. This corresponds to modulation indices $m=0.5 \%$ at $\lambda 6 \mathrm{~cm}$ and $m=1.2 \%$ at $\lambda 2.8 \mathrm{~cm}$, where $m[\%]=100 \sigma_{F} /\langle F\rangle$ is defined via the standard deviation of the flux density, $\sigma_{F}$, and its average value in time, $\langle F\rangle$. 


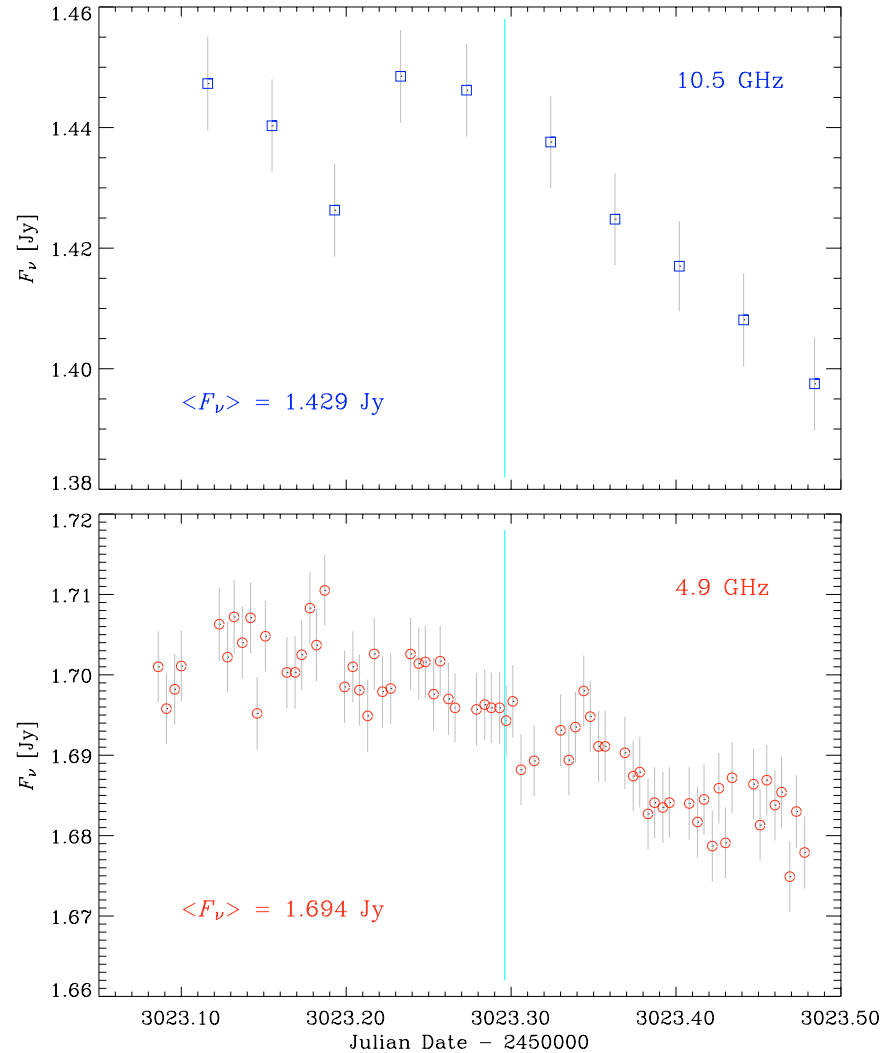

Fig. 7. Radio light curves at $\lambda 2.8 \mathrm{~cm}$ (top) and $\lambda 6 \mathrm{~cm}$ (bottom) obtained with the $100 \mathrm{~m}$ radio telescope at Effelsberg on January 18, 2004. The vertical line marks the start of the XMM-Newton observations.

A higher modulation index at shorter wavelengths, as found here, is not expected if interstellar scintillation were the physical mechanism responsible for the IDV activity (e.g. Rickett 1986). This motivates the determination of formal brightness temperatures and Doppler factors from the assumption that the flux variations have a source-intrinsic origin. During the observation the flux density of AO $0235+16$ decreased by $\sim 30 \mathrm{mJy}$ in $\sim 7 \mathrm{~h}$ at $\lambda 6 \mathrm{~cm}$, and by $\sim 50 \mathrm{mJy}$ in $\sim 6 \mathrm{~h}$ at $\lambda 2.8 \mathrm{~cm}$. Both these variations set the same lower limit to the source brightness temperature ${ }^{4}, T_{\mathrm{b}} \gtrsim 10^{17} \mathrm{~K}$, and to the Doppler factor, $\delta \gtrsim 46$, if the excess over the inverse-Compton limit of $10^{12} \mathrm{~K}$ is solely attributed to Doppler boosting.

\subsection{Optical observations}

At the time of the XMM-Newton pointing, AO 0235+16 was observable only for few hours after sunset in the optical band from the typical latitudes of WEBT observers. Besides this unfavourable situation, weather conditions on January 18-19 were bad over most of the northern hemisphere, so only a few WEBT collaborators could observe. Moreover, the source was in one of its faintest states, and this prevented most of the small telescopes from obtaining utilizable data.

\footnotetext{
${ }^{4}$ We adopt a flat cosmology with $H_{0}=71 \mathrm{~km} \mathrm{~s}^{-1} \mathrm{Mpc}^{-1}$ and $\Omega=$ 0.27 , which yields a source distance of $\sim 6140 \mathrm{Mpc}$.
}

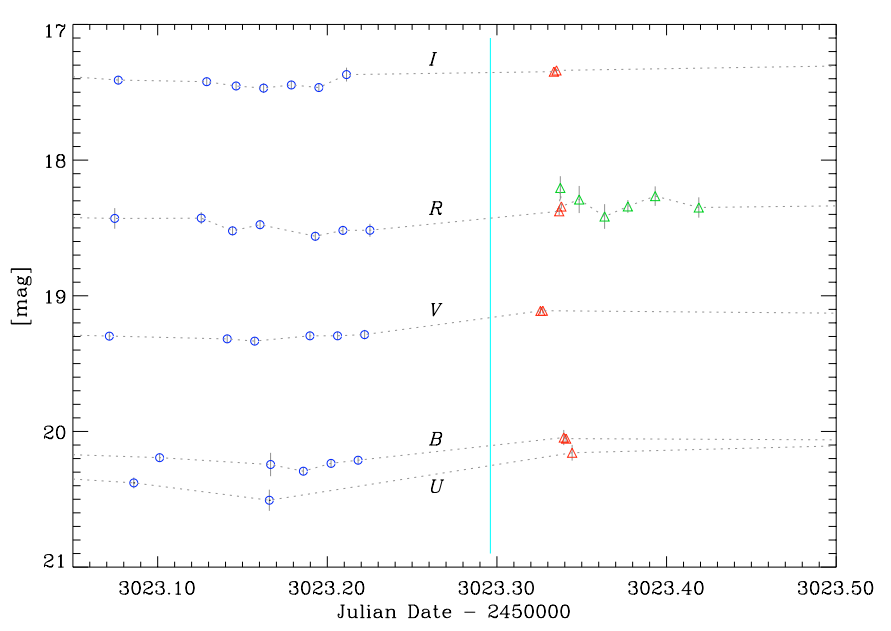

Fig. 8. UBVRI light curves obtained at Mt. Maidanak (blue circles) and at La Palma by the NOT (red triangles) and by the KVA (green triangles) on January 18, 2004. The vertical line marks the start of the XMM-Newton observations.

Table 3. Optical Monitor observation log during the AO $0235+16$ pointing.

\begin{tabular}{lccc}
\hline \hline Filter & Exp. (s) & Start & End \\
\hline$V$ & 2500 & Jan. 18, 19:15:29 & Jan. 18, 19:57:09 \\
$U$ & 2501 & Jan. 18, 20:02:17 & Jan. 18, 20:43:58 \\
$B$ & 2660 & Jan. 18, 20:49:03 & Jan. 18, 21:33:23 \\
$W 1$ & 4999 & Jan. 18, 21:38:50 & Jan. 18, 23:02:09 \\
$M 2$ & 4998 & Jan. 18, 23:07:18 & Jan. 19, 00:30:36 \\
$W 2$ & 5001 & Jan. 19, 00:35:43 & Jan. 19, 01:59:04 \\
Grism1 & 5000 & Jan. 19, 02:04:10 & Jan. 19, 03:27:30 \\
\hline
\end{tabular}

The UBVRI light curves obtained on January 18-19, 2004 are shown in Fig. 8. The observations started with the $150 \mathrm{~cm}$ telescope on Mt. Maidanak and then frames were taken in La Palma simultaneously with the XMM-Newton pointing by the $256 \mathrm{~cm}$ NOT and $35 \mathrm{~cm}$ KVA telescopes. However, the data uncertainties and poor sampling prevent one to recognize clear variability trends to be compared with radio and X-ray observations.

\subsection{Results from the Optical Monitor (OM)}

Besides the X-ray detectors, XMM-Newton also has a coaligned $30 \mathrm{~cm}$ optical-UV telescope, the Optical Monitor (OM). The instrument is equipped with optical $V B U$ filters plus ultraviolet UVW1 $\left(\lambda_{\mathrm{eff}}=291 \mathrm{~nm}\right), \operatorname{UVM} 2\left(\lambda_{\mathrm{eff}}=231 \mathrm{~nm}\right)$, and UVW2 $\left(\lambda_{\mathrm{eff}}=212 \mathrm{~nm}\right)$ ones.

The OM observation log during the XMM-Newton pointing of AO $0235+16$ on January $18-19,2004$ is shown in Table 3.

The pipeline products (PP) give the calibrated magnitudes calculated as $m=m_{0}-2.5 \log$ (counts $\left./ \mathrm{s}\right)$, where $m_{0}$ are the zeropoints in the various filters. They are continuously updated as more data are becoming available. As expected, the UV zeropoints are the most unstable ones. The zeropoints used for the present observations are reported in Table 4. 
Table 4. Results from the Optical Monitor observations of January 18-19, 2004; $m_{0}$ are the zeropoints.

\begin{tabular}{lcccccc}
\hline \hline & $V$ & $B$ & $U$ & $W 1$ & $M 2$ & 14.862 \\
\hline$m_{0}$ & 17.963 & 19.266 & 18.259 & 17.297 & 15.810 & $15.838(0.019)$ \\
\hline Star 1 & $13.100(0.002)$ & $13.689(0.001)$ & $13.582(0.002)$ & $14.217(0.004)$ & $16.010(0.037)$ \\
Star 2 & $12.784(0.002)$ & $13.649(0.001)$ & $13.944(0.003)$ & $14.804(0.005)$ & $17.563(0.057)$ & $17.395(0.101)$ \\
Star 3 & $12.984(0.002)$ & $13.761(0.001)$ & $13.927(0.003)$ & $14.776(0.005)$ & $17.353(0.049)$ & $17.226(0.089)$ \\
Star 6 & $14.056(0.004)$ & $14.776(0.003)$ & $14.741(0.005)$ & $15.463(0.008)$ & $17.487(0.054)$ & $17.613(0.122)$ \\
Star C1 & $14.848(0.007)$ & $15.868(0.006)$ & $16.488(0.014)$ & $17.419(0.027)$ & - & - \\
\hline AO 0235+16 & $19.22(0.07)$ & $20.09(0.07)$ & $20.12(0.10)$ & $19.95(0.11)$ & $19.68(0.22)$ & - \\
\hline
\end{tabular}

In order to check the reliability of the PP photometry, we report in Table 4 the OM PP magnitudes obtained for the reference stars in the field of $\mathrm{AO} 0235+16$. We notice that the PP $U B V$ magnitudes for the bright Stars 123 are close to the Smith et al. (1985) values, while the PP $B V$ photometry for the fainter Stars 6 and $C 1$ gives magnitudes larger than those obtained through ground-based observations (Fiorucci et al. 1998; González-Pérez et al. 2001).

In order to avoid the inclusion of the ELISA contribution, we reduced the OM frames with the IRAF "phot" routine, using an aperture radius of 2 pixels, corresponding to 0.953 arcsec. The results, obtained by differential photometry with respect to the reference stars and magnitudes listed in Table 4, are shown in the last row of the same table; the $V B U$ magnitudes are in satisfactory agreement with those derived from the NOT frames $(V=19.11 \pm 0.02, B=20.05 \pm 0.03, U=20.16 \pm 0.06)$.

\section{Broad-band spectral energy distribution}

Fitting the intrinsic spectral energy distributions (SEDs) of a blazar is the only way to test the capability of a model to interpret its broad-band emission. Since blazars are variable sources, SEDs must be constructed with simultaneous data; moreover, in order to convert observed magnitudes into intrinsic fluxes, extinction must be taken into account.

In the case of $\mathrm{AO} 0235+16$, a correct estimate of extinction is not an easy task. The NASA Extragalactic Database (NED) provides Galactic extinction coefficients for optical and near-IR bands. Galactic extinction for other bands can be derived following e.g. Cardelli et al. (1989), who give extinction laws from far-IR to far-UV as a function of wavelength and of the parameter $R_{V}=A_{V} / E(B-V)$, where $A_{V}$ is the extinction expressed in magnitudes in the $V$ band, and $E(B-V)$ is the extinction difference between the $B$ and $V$ bands. The mean value for the diffuse Galactic interstellar medium is $R_{V}=3.1$. Column 2 of Table 5 reports the Galactic extinction values given by NED for the UBVRIJHK bands plus the values obtained from Cardelli et al. (1989) for the OM $W 1, M 2$, and $W 2$ filters $^{5}$, setting $R_{V}=3.1$. Notice that, according to NED, $E(B-V)=0.079$.

\footnotetext{
${ }^{5}$ Had we used the more recent prescriptions by Fitzpatrick (1999) in the ultraviolet, we would have obtained a bit lower extinction corrections: $0.453,0.694$, and $0.763 \mathrm{mag}$ for the $W 1, M 2$, and $W 2$ bands, respectively.
}

Table 5. Extinction values (mag) for the Galaxy and the $z=0.524 \mathrm{ab}-$ sorber. The "NED" case adopts the UBVRIJHK values given by the Nasa Extragalactic Database plus the UV values calculated according to Cardelli et al. (1989) with $R_{V}=3.1$. The "Jun" case refers to the Junkkarinen et al. (2004) prescriptions for the Galactic as well as the $z=0.524$ absorptions. The last column shows the total extinction in the "Jun" case.

\begin{tabular}{lcccc}
\hline \hline Filter & $\begin{array}{c}A_{\lambda}^{\text {NED }} \\
z=0\end{array}$ & $\begin{array}{c}A_{\lambda}^{\text {Jun }} \\
z=0\end{array}$ & $\begin{array}{c}A_{\lambda}^{\text {Jun }} \\
z=0.524\end{array}$ & $\begin{array}{c}A_{\lambda}^{\text {Jun }} \\
\text { Total }\end{array}$ \\
\hline$W 2$ & 0.830 & 1.512 & 2.125 & 3.637 \\
$M 2$ & 0.741 & 1.350 & 1.938 & 3.288 \\
$W 1$ & 0.490 & 0.893 & 1.884 & 2.777 \\
$U$ & 0.430 & 0.742 & 1.777 & 2.519 \\
$B$ & 0.341 & 0.636 & 1.268 & 1.904 \\
$V$ & 0.262 & 0.482 & 0.991 & 1.473 \\
$R$ & 0.211 & 0.402 & 0.858 & 1.260 \\
$I$ & 0.153 & 0.287 & 0.615 & 0.902 \\
$J$ & 0.071 & 0.140 & 0.318 & 0.458 \\
$H$ & 0.046 & 0.088 & 0.187 & 0.275 \\
$K$ & 0.029 & 0.055 & 0.116 & 0.171 \\
\hline
\end{tabular}

However, we have already discussed the noticeable extraabsorption affecting the soft part of the X-ray spectrum, likely due to the $z=0.524$ absorber. This absorber should affect also the UV-optical-IR spectral region. The extinction law for this absorber is not directly known, but a possible recipe can be found in Junkkarinen et al. (2004). These authors performed a number of fits to an HST/STIS spectrum of AO $0235+16$ extending from far-UV to near-IR (1570-10266 ̊). They assumed that the intrinsic spectrum is a power law (or a broken power law) and modelled the Galactic extinction according to Cardelli et al. (1989) with $R_{V}=3.1$ and $E(B-V)=0.154$. As for the $z=0.524$ absorber, they tried different prescriptions, including the Cardelli et al. (1989) laws for the Galaxy with $R_{V}$ as a free parameter and the Pei (1992) laws for the Large and Small Magellanic Clouds. They concluded that the best fit is obtained when considering the Cardelli et al. (1989) laws with $R_{V}=2.5$ and $E(B-V)=0.23$. They warned that the fit is not perfect; deviations occur for $\lambda(z=0)>8700 \AA$ and $\lambda(z=$ $0)<2500 \AA$; in particular, this recipe leads to an overestimate of the extinction in the far-UV. Table 5 reports the Galactic extinction values following Junkkarinen et al. (2004): when compared with the "NED" case, the "Jun" case for $z=0$ (Col. 3) 


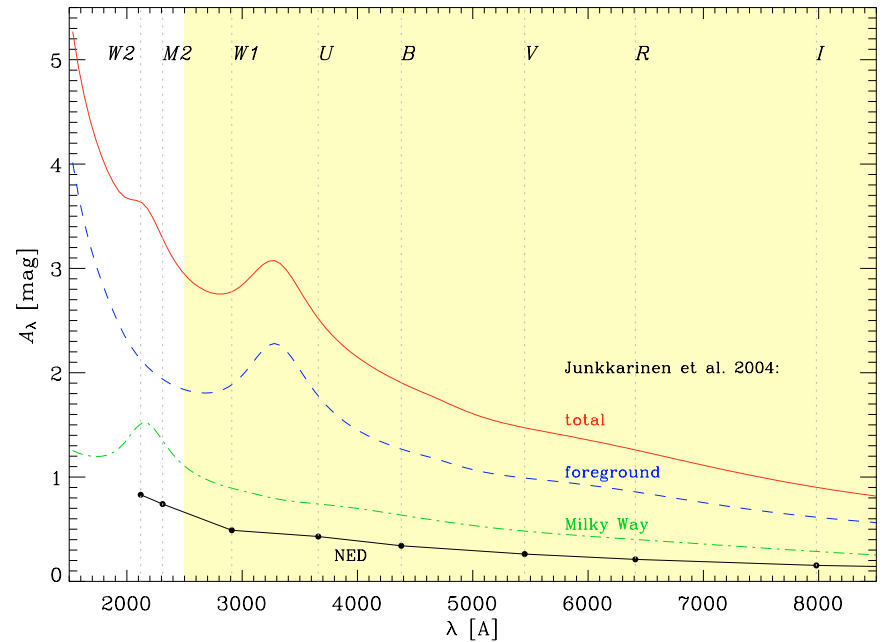

Fig. 9. The amount of absorption (mag) towards $A O 0235+16$ as a function of wavelength. The "NED" case is compared with the "Jun" one (see text); in this latter case, the total contribution is shown (red, solid line), together with the single contributions by the Milky Way (green, dashed-dotted) and by the $z=0.524$ foreground galaxy (blue, dashed). The yellow area indicates the wavelength region where the Junkkarinen et al. (2004) prescriptions are believed to give a good evaluation of the extinction.

implies an almost twice absorption; moreover, the absorption at $z=0.524$ (Col. 4) appears much stronger than the Galactic one. The total extinction to apply for de-reddening the source magnitudes in the various bands according to Junkkarinen et al. (2004) is given in the last column of Table 5. The same information is plotted in Fig. 9: the amount of absorbtion as a function of wavelength foreseen by the "NED" case (black points) is compared with the Junkkarinen et al. (2004) prescriptions for the Milky Way (green, dashed-dotted line), for the $z=0.524$ foreground galaxy (blue, dashed line), and for the total extinction due to both components (red, solid line). The yellow area highlights the frequency range where these prescriptions lead to a good fit of the HST/STIS spectrum.

Figure 10 shows the SED in the optical-UV frequency range during the XMM-Newton pointing of January 18-19, 2004. Optical data in UBVRI from the NOT are simultaneous with the optical $V B U$ and ultraviolet $W 1, M 2$ data from the OM.

De-reddened ground-based optical magnitudes from the NOT were converted into fluxes by using the zero-mag fluxes by Bessell et al. (1998). These data are shown as (empty and filled) circles in Fig. 10. The same was done for the $V B U$ data from the OM; as for the UV bands, fluxes were obtained using Vega as a reference (see OM documentation at http://xmm.vilspa.esa.es). The OM points are shown as (red) triangles.

In the figure empty symbols represent data obtained by de-reddening for the Galaxy extinction according to the "NED" case. Filled symbols indicate the unabsorbed optical-UV SED when both the Galactic and the $z=0.524 \mathrm{ab}-$ sorptions are taken into account following the prescriptions by Junkkarinen et al. (2004).

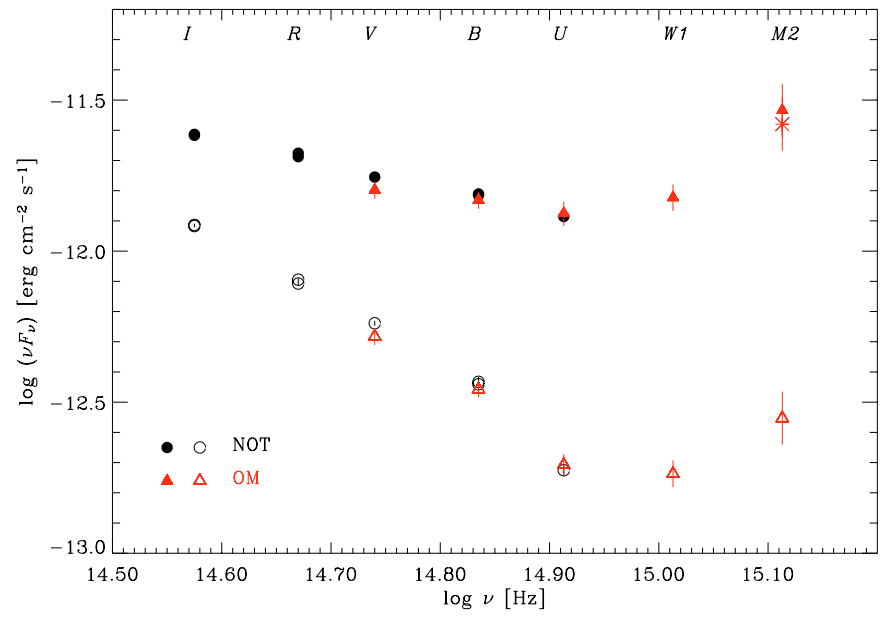

Fig. 10. Spectral energy distribution of AO $0235+16$ in the optical-UV frequency range during the XMM-Newton pointing of January 18-19, 2004. Empty and filled circles indicate IRVBU data from the NOT simultaneous with the XMM-Newton observations; triangles refer to the data from the XMM-Newton Optical Monitor (OM). Empty symbols represent data obtained according to the "NED" case for the Galactic extinction; filled symbols refer to the "Jun" case for the Galactic as well as the $z=0.524$ absorptions. The asterisk corresponds to a "corrected" "Jun" case (see text).

As one can see, when considering also the effect of the $z=0.524$ absorber, the optical spectral index of AO $0235+16$ becomes much smaller (from $\alpha=3.23$ to $\alpha=1.79$ according to the NOT data), and more similar to that of a typical lowenergy peaked BL Lac object. But the most striking feature is the SED hardening in the ultraviolet, present in both cases.

We warn about use of the $M 2$ flux, since it falls in the "uncertain" region of Fig. 9, and, moreover, the calibration of these UV data is also uncertain. Nevertheless, the fact that also in the "NED" case we see a dramatic slope change suggests that some slope variation indeed occurs, even if it may be less pronounced. On the other hand, if we try to quantify the possible overestimate of the absorption in the $M 2$ region from the model of Junkkarinen et al. (2004), we find a $\sim 10 \%$ excess on the flux, which would lower the point at the position marked with an asterisk in Fig. 10, and would represent only a slight change with respect to the previous situation.

Figure 11 shows the broad-band SED obtained by plotting simultaneous optical-UV-X-ray data from January 18-19, 2004 and contemporaneous or quasi-contemporaneous radio data. The optical and UV data are those shown in Fig. 10 ("Jun" case); the X-ray spectrum comes from the Model 2 fit to the three EPIC detectors together (see Sect. 3.1), taking into account the uncertainties on both the normalization and spectral index. Radio data at 4.9 and $10.5 \mathrm{GHz}$ are from simultaneous observations at Effelsberg (filled blue diamonds); upper and lower limits of the variation range during the XMM-Newton pointing have been indicated. As for the other radio frequencies (empty blue diamonds), the pairs of plotted values correspond to the data immediately preceding and following the XMM-Newton pointing (see Fig. 4). In Fig. 11 we have also plotted fictitious $J H K$ data (squares) calculated by adopting the optical-NIR colour indices obtained from NOT data at a 


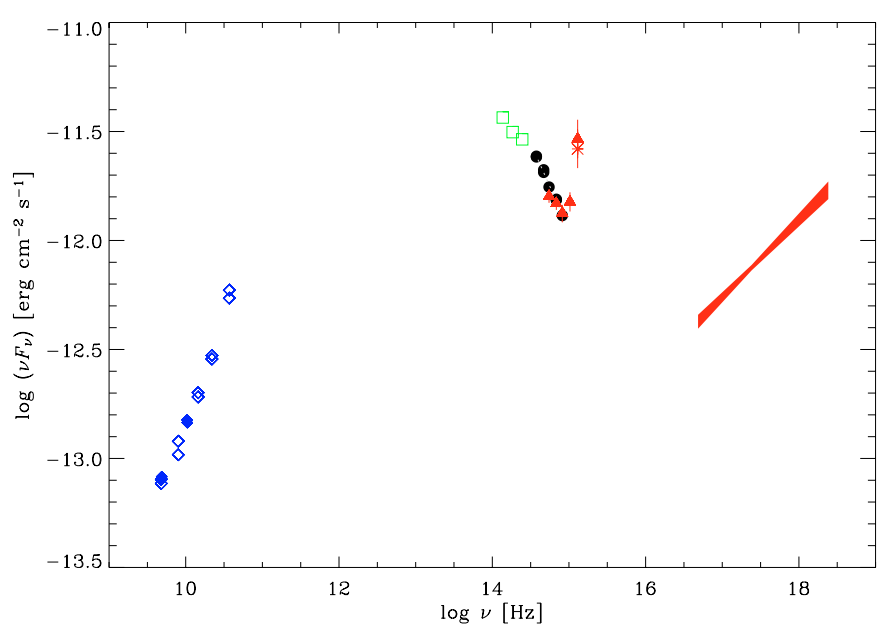

Fig. 11. Broad-band spectral energy distribution of AO $0235+16$ during the XMM-Newton pointing of January 18-19, 2004. Filled symbols indicate simultaneous data (see text for details).

similar brightness level. They have been de-reddened by using the extinction values shown in the last column of Table 5, i.e. the same "Jun" case adopted for the optical-UV bands, even if this spectral region is outside the HST/STIS spectrum used by Junkkarinen et al. (2004) to derive their model.

\section{Cross-correlations and time delays}

The existence of a correlation between the radio and optical fluxes of AO $0235+16$ was discussed in several papers; simultaneous radio and optical variability was found during the optical outbursts occurred in 1975, 1979, and 1997 (MacLeod et al. 1976; Ledden et al. 1976; Rieke et al. 1976; Balonek \& Dent 1980; Webb et al. 2000).

Clements et al. (1995) analysed time-extended optical and radio light curves, finding a general delay of the radio events with respect to the optical ones of 0-2 months. A correlation between the general trends in the two bands was noticed by Takalo et al. (1998). Raiteri et al. (2001) compared 25 years of optical and radio data and pointed out how in some cases optical outbursts seem to be simultaneous with the radio ones, while in other cases they lead the radio events. They suggested that this can be the signature of two different variability mechanisms.

Moreover, while Clements et al. (1995) did not find any time delay between variations at different radio frequencies, other authors (O'Dell et al. 1988; Raiteri et al. 2001) noticed that the changes observed at the lower frequencies lag the higher-frequency ones.

In the following we re-examine the matter by means of the Discrete Correlation Function (DCF; Edelson \& Krolik 1988; Hufnagel \& Bregman 1992; Peterson et al. 2004), a method conceived to analyze unevenly sampled data trains. For the optical data, we used de-reddened fluxes obtained according to the Junkkarinen et al. (2004) prescriptions (see previous section). Before calculating the DCF we binned the datasets in order to smooth the intraday features in the optical data and thus obtain a similar sampling in the radio and optical bands.
Table 6. Results of the DCF analysis on the optical ( $R$-band) and radio light curves. Time lags derived from both the peak $\left(\tau_{\mathrm{p}}\right)$ and the centroid $\left(\tau_{\mathrm{c}}\right)$ are given.

\begin{tabular}{lccc}
\hline \hline Bands & $\tau_{\mathrm{p}}$ (days) & $\mathrm{DCF}_{\mathrm{p}}$ & $\tau_{\mathrm{c}}($ days $)$ \\
\hline$R-37 \mathrm{GHz}$ & 30 & 0.4 & $30-35$ \\
$R-22 \mathrm{GHz}$ & 30 & 0.8 & $35-39$ \\
$R-14.5 \mathrm{GHz}$ & 30 & 0.6 & $45-50$ \\
$R-8 \mathrm{GHz}$ & 0,60 & $0.6,0.7$ & $-10,60-69$ \\
$R-5 \mathrm{GHz}$ & 80 & 0.5 & $65-70$ \\
\hline
\end{tabular}

We tried several bin sizes, from 1 to 10 days, to check how the results are sensitive to this smoothing procedure.

The DCF run on the new optical and radio data presented in this paper does not show significant peaks, meaning no radiooptical correlation over the last four observing seasons, and confirming what we noticed in Sect. 2.3 after a visual inspection of Fig. 4.

In contrast, a correlation is found when considering the whole datasets, including the data presented in Raiteri et al. (2001), optical data from the Hamburg Quasar Monitoring program (partially published by Schramm et al. 1994) and from CASLEO (Romero et al. 2002), the radio data published by Venturi et al. (2001), and the old radio data taken with RATAN (partially published by Kovalev et al. 1999b and Kiikov et al. 2002).

In particular, time delays between variations in the best sampled $R$-band light curve and those in the radio data can now be specified to a higher degree of detail. Table 6 summarizes the results of cross-correlation analysis when the light curves are binned over 2 days before calculating the DCF; time lags derived from both the peak, $\tau_{\mathrm{p}}$, and the centroid, $\tau_{\mathrm{c}}$, of the DCF are given, where centroids are obtained by considering all points with $\mathrm{DCF}>k \mathrm{DCF}_{\mathrm{p}}, \mathrm{DCF}_{\mathrm{p}}$ being the $\mathrm{DCF}$ value of the peak and $k$ ranging from 0.75 to 0.85 . From the $\tau_{\mathrm{c}}$ values shown in the table, one can see that the radio lag seems to increase with decreasing frequency. Notice that when considering the best-sampled radio light curve at $8 \mathrm{GHz}$ (see Fig. 12), another important peak appears at zero time lag, possibly suggesting that some of the radio and optical variations can indeed be simultaneous, as found in some previous studies.

Consistent estimates of delays are also derived from crosscorrelating radio datasets among themselves: taking the $8 \mathrm{GHz}$ light curve as the reference one, its variations seem to follow those seen at 37,22 , and $14.5 \mathrm{GHz}$ by $24-35,29-38$, and 14-15 days, respectively, and to lead those at $5 \mathrm{GHz}$ by 10-14 days. These results are similar to those recently found for other two blazars: S5 0716+71 (Raiteri et al. 2003) and BL Lac (Villata et al. 2004b).

The conclusion of the above analysis is that the radiooptical correlation comes mainly from the major events, which are seen first in the optical band and then progressively later in the lower-frequency bands, while during faint states the radio and optical radiations behave in a different way. 


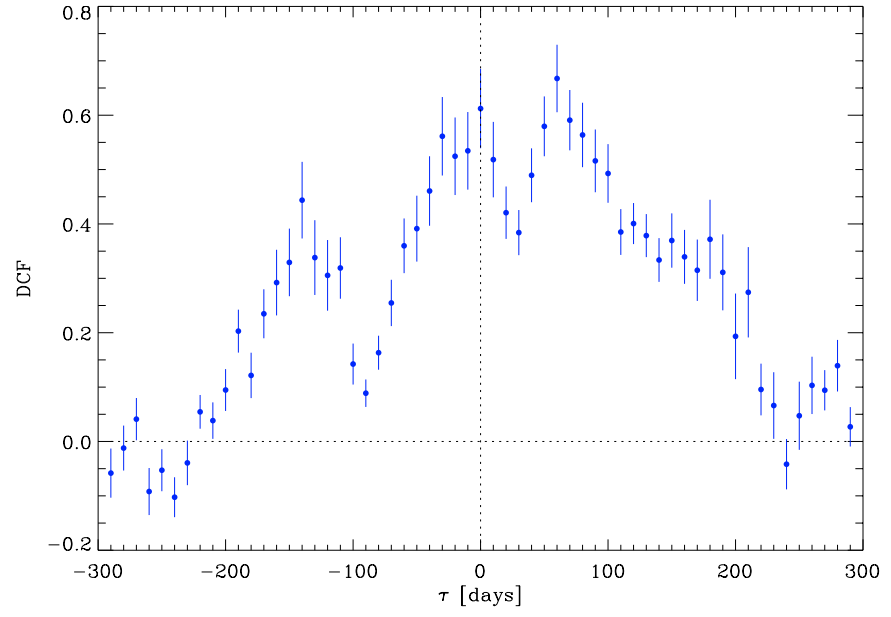

Fig. 12. Discrete correlation function (DFC) between the $R$-band and $8 \mathrm{GHz}$ light curves. Two major peaks are visible, corresponding to time lags of 0 and 60 days.

\section{Discussion and conclusions}

A huge international observing effort led by the WEBT collaboration was undertaken to investigate the emission behaviour of the BL Lac object AO 0235+16 during the observing season 2003-2004. Observations were carried out at different frequencies, from the radio band to X-ray energies. Notwithstanding the faintness of the source, which made observations rather difficult, we obtained an unprecedented monitoring density in the optical and near-IR bands.

The source was fairly active in the optical and near-IR bands, but rather "quiet" at the radio frequencies. In particular, the radio flux base level is higher at the lower frequencies. While the optical and near-IR variations seem to be well correlated, no correlation is seen between the optical-NIR and radio behaviours. However, this source has shown correlated optical and radio outbursts in the past, with short time delays, repeating quasi-periodically every $5.7 \pm 0.5$ years (Raiteri et al. 2001). Moreover, in a previous study on the optical and radio behaviour of this source, Takalo et al. (1998) noticed how the radio variability in general follows the optical behaviour, even if some optical events have no radio counterpart. Also during the 2003-2004 observing season the main trend seems to be optical activity not significantly affecting the radio band. In other words, the correlation between radio and optical variations found in previous studies concerns the major outbursts only.

This situation suggests that different variability mechanisms are at work. As already noticed for other blazars (e.g. BL Lac, Villata et al. 2004a,b; S5 0716+71, Raiteri et al. 2003), long-term variability can be ascribed to Doppler factor variations (of possible geometrical origin), while faster flares are most likely of an intrinsic nature. In the case of AO $0235+16$ it has been shown (Ostorero et al. 2004; see also Romero et al. 2003) that a rotating helical jet generated by a binary black hole system can account for the periodic behaviour of radio and correlated optical outbursts. Another scenario suggests that contemporaneous radio and optical outbursts are the consequences of microlensing due to intervening objects (Stickel et al. 1988; but see Kayser 1988, for a critical discussion). On the other hand, the "inter-outbursts" strong optical activity with no radio counterpart would imply that the optically emitting region is spatially distinct from the radio emitting one. In summary, the two emitting regions should be distant enough to provide uncorrelated intrinsic variations, but close enough to allow a quasi-simultaneous beaming of both of them as the helical jet rotates.

The radio (and optical) outburst predicted to peak in February-March 2004 (with a $~ 6$ month uncertainty) has not yet occurred. The WEBT campaign is still ongoing and will cover the entire 2004-2005 observing season.

The XMM-Newton pointing of January 18-19, 2004 found the source in a very faint state. Nevertheless, the X-ray spectrum is very well determined in both slope and intensity: it is well fitted by a single power law with extra-absorption. Its hardness suggests an inverse-Compton origin of the observed $\mathrm{X}$-ray emission. No significant variability was seen in the X-ray light curve, while a decreasing trend (about $2-3 \%$ in $6-7 \mathrm{~h}$ ) is visible in the contemporaneous Effelsberg radio data. This variation, if intrinsic, would imply a brightness temperature well exceeding the Compton limit, and a corresponding Doppler factor $\delta \gtrsim 46$. As for the simultaneous optical data, their uncertainty and sampling do not allow one to infer any clear trend.

Our work highlights the role played by the source environment. On the one hand ELISA (the southern AGN) affects the optical-UV photometry of AO $0235+16$, especially when it is in a faint state and more in the bluer part of the spectrum. On the other hand, the eastern galaxy, possibly in conjunction with the ELISA host galaxy, acts as an absorber of the NIR-to-X-ray radiation. When the AO $0235+16$ broad-band spectral energy distribution is constructed with data corrected for both these effects, it suggests the existence of a bump in the UV spectral region. Indeed, when comparing their February 11, 1998 HST/STIS de-reddened spectrum with a contemporaneous ASCA X-ray spectrum, Junkkarinen et al. (2004) noticed that a smooth connection of the optical-UV data with the X-ray ones in the SED is not possible, but a bump in the UV-soft-X-ray energy range must be invoked. They considered this possibility unlikely, and suggested that the problem may lie in an incorrect modelling of the $z=0.524$ absorber extinction law. A more extended study of this problem through the examination of a number of broad-band SEDs of this source is under way (Raiteri et al. 2005).

Acknowledgements. Based on observations obtained with XMM-Newton, an ESA science mission with instruments and contributions directly funded by ESA Member States and NASA. Based on observations with the $100 \mathrm{~m}$ telescope of the MPIfR (Max-Planck-Institut für Radioastronomie) at Effelsberg. Based on observations made with the Nordic Optical Telescope, operated on the island of La Palma jointly by Denmark, Finland, Iceland, Norway, and Sweden, in the Spanish Observatorio del Roque de los Muchachos of the Instituto de Astrofísica de Canarias. Based on observations made with the Italian Telescopio Nazionale Galileo (TNG) operated on the island of La Palma by the Centro Galileo Galilei of the INAF (Istituto Nazionale di Astrofisica) at the Spanish Observatorio del Roque de los Muchachos of the Instituto de Astrofísica de Canarias. Based on observations taken at TIRGO 
(Gornergrat, Switzerland). TIRGO is operated by CAISMI-CNR Arcetri, Firenze, Italy. The Indian Astronomical Observartory at Hanle is operated by the Indian Institute of Astrophysics, Bangalore. This research has made use of data from the University of Michigan Radio Astronomy Observatory, which is supported by the National Science Foundation and by funds from the University of Michigan. RATAN-600 observations were partly supported by the Russian State Program "Astronomy" (project 1.2.5.1), the Russian Ministry of Education and Science, and the Russian Foundation for Basic Research (projects 01-02-16812 and 02-02-16305). This work was partly supported by the European Community's Human Potential Programme under contract HPRN-CT-2002-00321, by the Italian Space Agency (ASI) under contract CNR-ASI 1/R/073/02 and by the Italian MIUR under grant Cofin2001/028773. M.B. acknowledges support by NASA through XMM-Newton GO grant NNG 04GF70G. V. M. Larionov and V. A. Hagen-Thorn were supported by Russian Federal Program "Astronomy", project 40.022.1.1.1001. M.K. was supported for this research through a stipend from the International Max Planck Research School (IMPRS) for Radio and Infrared Astronomy at the University of Bonn. C.M.R. is grateful to E. Trussoni, A. Capetti, and J. Heidt for useful discussions.

\section{References}

Aller, M. F., Aller, H. D., Hughes, P. A., \& Latimer, G. E. 1999, ApJ, 512,601

Balonek, T. J., \& Dent, W. A. 1980, ApJ, 240, L3

Bessell, M. S., Castelli, F., \& Plez, B. 1998, A\&A, 333, 231

Burbidge, E. M., Beaver, E. A., Cohen, R. D., et al. 1996, AJ, 112, 2533

Cardelli, J. A., Clayton, G. C., \& Mathis, J. S. 1989, ApJ, 345, 245

Clements, S. D., Smith, A. G., Aller, H. D., \& Aller, M. F. 1995, AJ, 110,529

Cohen, R. D., Smith, H. E., Junkkarinen, V. T., \& Burbidge, E. M. 1987, ApJ, 318, 577

Comastri, A., Fossati, G., Ghisellini, G., \& Molendi, S. 1997, ApJ, 480,534

Dennett-Thorpe, J., \& de Bruyn, A. G. 2002, Nature, 415, 57

Edelson, R. A., \& Krolik, J. H. 1988, ApJ, 333, 646

Elvis, M., Lockman, F. J., \& Wilkes, B. 1989, AJ, 97, 777

Fiorucci, M., Tosti, G., \& Rizzi, N. 1998, PASP, 110, 105

Fitzpatrick, E. L. 1999, PASP, 111, 63

Frey, S., Gurvits, L. I., Altschuler, D. R., et al. 2000, PASJ, 52, 975

Fujisawa, K., Kobayashi, H., Wajima, K., et al. 1999, PASJ, 51, 537

Ghosh, K. K., \& Soundararajaperumal, S. 1995, ApJS, 100, 37

González-Pérez, J. N., Kidger, M. R., \& Martín-Luis, F. 2001, AJ, 122,2055

Heeschen, D. S., Krichbaum, T., Schalinski, C. J., \& Witzel, A. 1987, AJ, 94, 1493

Heidt, J., \& Wagner, S. J. 1996, A\&A, 305, 42

Hufnagel, B. R., \& Bregman, J. N. 1992, ApJ, 386, 473

Jauncey, D. L., \& Macquart, J.-P. 2001, A\&A, 370, L9

Jones, T. W., O’Dell, S. L., \& Stein, W. A. 1974, ApJ, 192, 261

Jorstad, S. G., Marscher, A. P., Mattox, J. R., et al. 2001, ApJS, 134, 181

Junkkarinen, V. T., Cohen, R. D., Beaver, E. A., et al. 2004, ApJ, 614, 658

Kadler, M., et al. 2005, in preparation

Kayser, R. 1988, A\&A, 206, L8

Kedziora-Chudczer, L., Jauncey, D. L., Wieringa, M. H., Tzioumis, A. K., \& Reynolds, J. 2001, MNRAS, 325, 1411

Kiikov, S. O., Mingaliev, M. G., Stolyarov, V. A., \& Stupalov, M. S. 2002, Bull. Spec. Astrophys. Obs., 54, 5
Kovalev, Y. Y., Nizhelsky, N. A., Kovalev, Yu. A., et al. 1999a, A\&AS, 139,545

Kovalev, Y. Y., Teräsranta, H., Tornikoski, M., \& Valtaoja, E. 1999b, in BL Lac Phenomenon, ed. L. O. Takalo, \& A. Sillanpää (San Francisco: ASP), ASP Conf. Ser., 159, 65

Kraus, A., Quirrenbach, A., Lobanov, A. P., et al. 1999, A\&A, 344, 807

Kraus, A., Krichbaum, T. P., Wegner, R., et al. 2003, A\&A, 401, 161

Ledden, J. E., Aller, H. D., \& Dent, W. A. 1976, Nature, 260, 752

Lovell, J. E. J., Jauncey, D. L., Bignall, H. E., et al. 2003, AJ, 126, 1699

MacLeod, J. M., Andrew, B. H., \& Harvey, G. A. 1976, Nature, 260, 751

Madejski, G., Takahashi, T., Tashiro, M., et al. 1996, ApJ, 459, 156

Nilsson, K., Charles, P. A., Pursimo, T., et al. 1996, A\&A, 314, 754

Noble, J. C., \& Miller, H. R. 1996, PASPC, 110, 30

O’Dell, S. L., Dennison, B., Broderick, J. J., et al. 1988, ApJ, 326, 668

Ostorero, L., Villata, M., \& Raiteri, C. M. 2004, A\&A, 419, 913

Ott, M., Witzel, A., Quirrenbach, A., et al. 1994, A\&A, 284, 331

Padovani, P., Costamante, L., Giommi, P., et al. 2004, MNRAS, 347, 1282

Pei, Y. C. 1992, ApJ, 395, 130

Peterson, B. M., Ferrarese, L., Gilbert, K. M., et al. 2004, ApJ, 613, 682

Quirrenbach, A., Witzel, A., Krichbaum, T. P., et al. 1992, A\&A, 258, 279

Raiteri, C. M., Villata, M., Aller, H. D., et al. 2001, A\&A, 377, 396

Raiteri, C. M., Villata, M., Tosti, G., et al. 2003, A\&A, 402, 151

Raiteri, C. M., et al. 2005, A\&A, in preparation

Rickett, B. J. 1986, ApJ, 307, 564

Rickett, B. J., Witzel, A., Kraus, A., Krichbaum, T. P., \& Qian, S. J. 2001, ApJ, 550, L11

Rieke, G. H., Grasdalen, G. L., Kinman, T. D., et al. 1976, Nature, 260, 754

Romero, G. E., Combi, J. A., Benaglia, P., et al. 1997, A\&A, 326, 77

Romero, G. E., Cellone, S. A., \& Combi, J. A. 2000, A\&A, 360, L47

Romero, G. E., Cellone, S. A., Combi, J. A., \& Andruchow, I. 2002, A\&A, 390, 431

Romero, G. E., Fan, J.-H., \& Nuza, S. E. 2003, ChJAA, 3, 513

Schramm, K.-J., Borgeest, U., von Linde, J., Linnert, M. D., \& Schramm, T. 1994, A\&AS, 106, 349

Smith, P. S., Balonek, T. J., Heckert, P. A., Elston, R., \& Schmidt, G. D. 1985 , AJ, 90, 1184

Stickel, M., Fried, J. W., \& Kühr, H. 1988, A\&A, 198, L13

Takalo, L. O., Kidger, M. R., de Diego, J. A., et al. 1992, AJ, 104, 40

Takalo, L. O., Sillanpää, A., Valtaoja, E., et al. 1998, A\&AS, 129, 577

Teräsranta, H., Tornikoski, M., Mujunen, A., et al. 1998, A\&AS, 132, 305

Venturi, T., Dallacasa, D., Orfei, A., et al. 2001, A\&A, 379, 755

Villata, M., Mattox, J. R., Massaro, E., et al. 2000, A\&A, 363, 108

Villata, M., Raiteri, C. M., Kurtanidze, O. M., et al. 2002, A\&A, 390, 407

Villata, M., Raiteri, C. M., Kurtanidze, O. M., et al. 2004a, A\&A, 421, 103

Villata, M., Raiteri, C. M., Aller, H. D., et al. 2004b, A\&A, 424, 497

Wagner, S. J., \& Witzel, A. 1995, ARA\&A, 33, 163

Wagner, S. J., Witzel, A., Heidt, J., et al. 1996, AJ, 111, 2187

Webb, J. R., Howard, E., Benítez, E., et al. 2000, AJ, 120, 41

Wiik, K., et al. 2005, A\&A, in preparation

Witzel, A., Heeschen, D. S., Schalinski, C., \& Krichbaum, Th. 1986, Mitt. Astron. Ges, 65, 239

Worrall, D. M., \& Wilkes, B. J. 1990, ApJ, 360, 396

Yanny, B., York, D. G., \& Gallagher, J. S. 1989, ApJ, 338, 735 